Saudi Journal of Medicine

Abbreviated Key Title: Saudi J Med ISSN 2518-3389 (Print) |ISSN 2518-3397 (Online) Scholars Middle East Publishers, Dubai, United Arab Emirates

Journal homepage: https://saudijournals.com/sjm

Original Research Article

\title{
Effects of Different Ligation Systems on Arch Expansion, Incisor Inclination and Treatement Duration in Patients with Mild to Moderate Crowding Patients- A Prospective Study
}

Dr. Anshu Singh MDS $^{1 *}$, Dr. Hitesh Ramdas Sawant ${ }^{2}$, Dr. Ravi Jhamb ${ }^{3}$, Dr. Kritika Sehrawat ${ }^{4}$, Dr. Vikranth Shetty MDS $^{5}$, Dr. Dhananjay Rathod ${ }^{6}$

\begin{abstract}
${ }^{1}$ Senior Lecturer, Department of Orthodontics, Awadh Dental College and Hospital, Jamshedpur, Jharkhand India
${ }^{2}$ PG Student, Dept of Orthodontics \& Dentofacial Orthopaedics, Bharati Vidyapeeth Deemed to be University Dental College and Hospital, Navi Mumbai India

${ }^{3}$ Assistant professor, Department of Orthodontics and Dentofacial Orthopeadics, Pacific Dental College and hospital, Debari, Udaipur, Rajasthan India

${ }^{4}$ Consultant Oral \& Maxillofacial Surgeon, BM Gupta Hospital, Uttam Nagar, New Delhi

${ }^{5}$ Professor, Department of Orthodontics, Tatyasaheb Kore Dental College and Research Centre, New Pargaon, Kolhapur, Maharashtra India

${ }^{6}$ Assistant professor, Department of Orthodontics, Hazaribagh College of Dental Sciences, Hazaribagh, Jharkhand India
\end{abstract}

DOI: $10.36348 /$ sjm.2020.v05i10.004

| Received: 09.10.2020 | Accepted: 17.10.2020 | Published: 21.10 .2020

*Corresponding Author: Dr. Anshu Singh MDS

\section{Abstract}

Background and objective: One of the greatest challenges for the orthodontist is to select the correct ligation system to get optimum results. Ligation method refers to the means by which an arch wire is held inside the bracket. Recently developed ligation methods have been designed and built directly to brackets. The purpose of this study was to evaluate the effects of different ligation systems on arch expansion, incisor inclination and with regard to treatement duration in patients with mild to moderate crowding in maxillary arch. Materials and methods: Sixty patients were selected from a pool of patients amenable to non-extraction treatment in maxillary arch and those who satisfied other inclusion criteria. Patients were randomly allocated to three different ligation systems with equal sample size of 20 each. The model cast were evaluated for assessing maxillary arch changes, incisor inclination, and arch expansion. Analysis of covariance (ANCOVA) was used to compare the influence of the 3 bracket systems. Results: We found that the type of bracket had sgnificant effect on many of the transverse dimensional changes in maxillary arch No statistical significant difference in incisor inclination changes. The use of passive or active self-ligating bracket reduces the treatment duration. Conclusion: Non extraction treatment of crowding in maxillary arch with different ligation methods showed that compared to conventional system use of self-ligation bracket might be mechanically more efficient and had greater magnitude of expansion.

Keywords: Ligation Systems Inclination Treatement Crowding Patients.

Copyright () 2020 The Author(s): This is an open-access article distributed under the terms of the Creative Commons Attribution 4.0 International License (CC BY-NC 4.0) which permits unrestricted use, distribution, and reproduction in any medium for non-commercial use provided the original author and source are credited.

\section{INTRODUCTION}

In current system of orthodontic practice, there are so many ways to treat moderate crowding on a nonextraction basis with considerable degree of success. To get optimum result, the choice of ligation system and type of bracket used are of important factors as this will provide efficient treatment mechanics. Currently, the three most common ligation systems are orthodontists-conventional elastic ligation and active and passive self-ligating (SL) brackets $[1,2]$. The necessity of these ligation types in arches with mild to moderate crowding is that it facilitates arch expansion and also bodily movement of teeth. The clinical efficacy of the self-ligating (SL) has gained popularity among many clinicians and it was developed to overcome shortcomings of conventional brackets. The concept of self-ligating system can be dated back to 1935 and it has evolved over the past 30 years [3]. Now it is conceptualized in variety of designs fabricated from different manufacturers. These brackets do not require a movable component to hold the wire in its place. There are some reports which suggested that conventional elastomeric ligation of brackets will aggravate periodontal health. Self-ligating brackets have been proved advantageous over conventional system which includes increased patient comfort due to the absence of ligatures; improved oral hygiene .To date there is only little literature which compares the clinical effectiveness and dimensional changes in maxillary arch. 
Hence the purpose of this prospective study were therefore to evaluate and assess the effects of different ligation systems on arch expansion,incisor inclination and treatment duration in patients with mild to moderate crowding in maxillary arch.

\section{MATERIALS AND METHODS}

After obtaining ethical approval from the Institutional ethical committee 60 subjects were recruited in to the study. The patients were randomly allocated in three groups (conventional bracket system ovation, Dentsply GAC International) $(n=20)$, Passive self-- ligation ((Damon Q), $(\mathrm{n}=20)$ and Active self-ligation (ASL-In-Ovation C) brackets $(n=20)$ with a computer-generated randomization program done by a statistician independent from the study. Impressions for study models were taken before the start of treatment and after treatment completion. Finally digital study models were generated. The major population of study were of class I malocclusion. All patients had maxillary constriction caused by a dental transverse discrepancy. Therefore, in all 3 groups, we applied broad arch wires all orthodontic investigators were given a treatment protocol. Models were scanned and changes in the variables like maxillary intecanine width, interfirst premolar width, intermolar widths, overbite, overjet after the commencement of treatment is recorded, .The duration of treatment were also addressed in the data collection. The results of three groups were analysed using Analysis of covariance (ANCOVA).

\section{INCLUSION CRITERIA}

1. No systemic illness, as reported by patients.

2. Not under any drug therapy.

3. Good oral hygiene and periodontal health

4. Cooperative and motivated.

5. In the permanent dentition with all teeth present at least to the first molars.
6. Maxillary arch crowding less than $6 \mathrm{~mm}$

\section{EXCLUSION CRITERIA}

1. Complex medical history

2. Patients who required surgery to correct skeletal discrepancies.

3. Patients with cleft lip,palate and craniofacial anomalies

4. Uncooperative patients.

\section{RESULTS}

The participants represented population of different demographic characteristics and the mean age of passive self-ligating group were $20.7 \pm 11.3$ years $(n=20)$, active self-ligating $23.12 \pm 14.03$ years and conventional group $20.08 \pm 14.3$ years. The majority of population were male. The measurements were obtained by scanning the model cast using orthoanalyzed software before and after treatment, their dimensional changes are plotted in Table1.

All 60 subjects completed the study, and data shows that transverse measurements has considerably increased post treatment in all three groups, but those does not show any statistical significance. Intercanine dimensions increased in all 3 groups with slightly greater value in the passive self-ligating group (Damon Q).This increase in value were found to be significant predictors of the maxillary arch expansion and alleviation of crowding in all groups in particular study period. Transverse expansion was more pronounced in intermolar area for all systems. Mean increase of 1.89 $\mathrm{mm}$ is seen in Damon Q group post treatment. The magnitude of increase is more in self-ligating group. When analyzed for changes in incisor inclination, no statistically significant difference between active and passive ligation group. The mean duration of treatment was shorter for self-ligation group.

\begin{tabular}{|l|l|l|l|}
\hline Pre treatment & Damon Q mean (SD) & In Ovation C mean (SD) & ovation N mean (SD) \\
\hline intercanine & $31.34(3.07)$ & $32.32(2.36)$ & $33.7(2.93)$ \\
\hline interpremolar & $38.37(3.04)$ & $38.90(3.64)$ & $39.48(4.02)$ \\
\hline intermolar & $47.27(3.85)$ & $49.28(4.07)$ & $48.07(3.06)$ \\
\hline Post treatment & & & \\
\hline intercanine & $34.64(1.07)$ & $34.52(2.4)$ & $34.57(1.33)$ \\
\hline interpremolar & $43.44(2.04)$ & $44.10(2.42)$ & $43.82(3.02)$ \\
\hline intermolar & $49.16(2.85)$ & $50.28(3.37)$ & $49.47(2.76)$ \\
\hline $\begin{array}{l}\text { Pretreatment maxillary incisor } \\
\text { inclination }\end{array}$ & $112.75(6.28)$ & $111.08(6.06)$ & $110.25(2.46)$ \\
\hline $\begin{array}{l}\text { Posttreatment maxillary incisor } \\
\text { inclination }\end{array}$ & $114.14(5.75)$ & $113.02(4.59)$ & $113.20(5.19)$ \\
\hline Treatment duration & 14.6 & 13.6 & 15.8 \\
\hline
\end{tabular}

\section{DISCUSSION}

Our study compared the changes in transverse dimensions of maxillaty arch, amount of arch expansin and change in incisor inclination in patients with moderate crowding treated with different ligation systems. All patients were treated with same protocol and torque prescription but using different bracket system. We found that all three groups gained increased maxillary transverse dimension post treatment and greater expansion was seen with the self-ligating brackets. Greater magniture of improvement in the intermolar width in all system, which is contrary to a 
study by Franchi L et al. [4].He reported expansion ranging from 1.71 to $3.65 \mathrm{~mm}$ for maxillary transverse dimensions with increases peaking in the premolar region. There was significant expansion of all variables in the transverse plane in our study.

Self-ligating brackets based on the arch wire interaction divided into passive, active and interactive self-ligating brackets. In passive type, the clip or rigid door does not actively press against the arch wire. This will specifically lower resistence to sliding and promote patient tolerance. Active system are usually able to maintain a large amount of contact with archwire and better torque control[5,6].Our study suggest that selfligating groups shows greater level of expansion than conventional. We observed more amount of intercanine width in passive self-ligating group. Damon wires have a broad arch shape, particularly in the buccal segments, and could have contributed to the amount of expansion reported. Our post - treatement intermolar width is more in Damon group than conventional group, which is consistent with other studies in literature $[7,8]$.

The findings of our study suggest that degree of incisor inclination showed no significant difference between the self-ligating and conventional groups. Cattaneo et al. compared active and passive selfligating brackets using cone-beam computed tomography scans also showed no significant difference [9] Therefore, the "lip bumper" effect of the Damon system could not be substantially claimed in our study findings [10].The technique by which the conventional ligation resolve crowding is more through an incisal proclination while self-ligation brackets relieve crowding by facilitating more passive space closure.

There are studies which claimed that selfligating bracket had a a significantly shorter mean arch wire ligation and removal times than conventional[11].Consequently our results also proved that Self ligation brackets were more efficient than conventional groups in terms of treatment time to alleviate crowding and achieve alignment.

\section{CONCLUSION}

Our study emphasizes the advantageous and efficiency of self-ligation brackets over convention bracket system in terms of maxillary expansion, transverse dimensional changes and treatment duration. Compared to conventional system use of self-ligation bracket in crowded maxillary arch might be mechanically simple and less cumbersome.

\section{REFERENCE}

1. Thomas, S., Sherriff, M., \& Birnie, D. (1998). A comparative in vitro study of the frictional characteristics of two types of self-ligating brackets and two types of pre-adjusted edgewise brackets tied with elastomeric ligatures. The European Journal of Orthodontics, 20(5), 589-596.

2. Franchi, L., Baccetti, T., Camporesi, M., \& Barbato, E. (2008). Forces released during sliding mechanics with passive self-ligating brackets or nonconventional elastomeric ligatures. American Journal of Orthodontics and Dentofacial Orthopedics, 133(1), 87-90.

3. Fleming, P. S., DiBiase, A. T., \& Lee, R. T. (2008). Self-ligating appliances: evolution or revolution?. Australian Orthodontic Journal, 24(1), 41.

4. Franchi, L., Baccetti, T., Camporesi, M., \& Lupoli, M. (2006). Maxillary arch changes during leveling and aligning with fixed appliances and low-friction ligatures. American journal of orthodontics and dentofacial orthopedics, 130(1), 88-91.

5. Harradine, N. W. T. (2003). Self-ligating brackets: where are we now?. Journal of Orthodontics, 30(3), 262-273.

6. Fleming, P. S., DiBiase, A. T., \& Lee, R. T. (2010). Randomized clinical trial of orthodontic treatment efficiency with self-ligating and conventional fixed orthodontic appliances. American Journal of Orthodontics and Dentofacial Orthopedics, 137(6), 738-742.

7. Vajaria, R., BeGole, E., Kusnoto, B., Galang, M. T., \& Obrez, A. (2011). Evaluation of incisor position and dental transverse dimensional changes using the Damon system. The Angle Orthodontist, 81(4), 647-652.

8. Pandis, N., Polychronopoulou, A., \& Eliades, T. (2007). Self-ligating vs conventional brackets in the treatment of mandibular crowding: a prospective clinical trial of treatment duration and dental effects. American Journal of Orthodontics and Dentofacial Orthopedics, 132(2), 208-215.

9. Cattaneo, P. M., Treccani, M., Carlsson, K., Thorgeirsson, T., Myrda, A., Cevidanes, L. H. S., \& Melsen, B. (2011). Transversal maxillary dento- alveolar changes in patients treated with active and passive self- ligating brackets: a randomized clinical trial using CBCT- scans and digital models. Orthodontics \& craniofacial research, 14(4), 222-233.

10. Damon, D. H. (1998). The Damon low-friction bracket; a biologically compatible straight-wire system. J Clin Orthod, 32, 670-680.

11. Paduano, S., Cioffi, I., Iodice, G., Rapuano, A., \& Silva, R. (2008). Time efficiency of self-ligating vs conventional brackets in orthodontics: effect of appliances and ligating systems. Progress in Orthodontics, 9(2), 74-80. 